Check for updates

Cite this: RSC Adv., 2018, 8, 19251

\title{
Solar salt doped by MWCNTs as a promising high thermal conductivity material for CSP
}

\author{
Yanze Wu, (D) abc Jinli Li, ${ }^{\text {*ab }}$ Min Wang, ${ }^{\text {*ab }}$ Huaiyou Wang, ${ }^{\text {ab }}$ Yuan Zhong, ${ }^{\text {ab }}$ \\ Youjing Zhao, ${ }^{\text {ab }}$ Ming Wei ${ }^{\text {ab }}$ and Yan Liab
}

Solar salt has great advantages in solar thermal power generation compared to other molten salts, but its thermal conductivity needs to be further improved. Multi-walled carbon nanotubes (MWCNTs) have excellent thermal properties that can improve the thermal conductivity of materials as additives. In this study, five kinds of solar salt/MWCNTs composites with different doping amounts were prepared by a high-temperature melting method. The results showed that doping with MWCNTs can indeed improve the thermal properties of solar salt. We studied their quantitative structure-activity relationship (QSAR) in order to explain these phenomena. According to the TG-DSC analysis, there was almost no change in the melting point and decomposition temperature; the XRD analysis revealed that the bulk of the material was still $\mathrm{NaNO}_{3}$ and $\mathrm{KNO}_{3}$, which did not change; and according to Archimedes' method, the density of the materials also changes little. The thermal conductivity of the material was measured by the laser flash method; the results showed that the thermal conductivity of the sample with $0.3 \%$ doping increased by $293 \%$, reaching $1.65 \mathrm{~W}(\mathrm{~m} \mathrm{~K})^{-1}$. XPS analysis showed that the MWCNTs were purified and the impurity groups were largely removed after high-temperature melting. From the laser Raman analysis, the $V_{3}$ frequency peak of the sample with $0.3 \%$ doping was red-shifted, and for the other samples was blue-shifted. The SEM images showed that the sample with $0.3 \%$ doping was the most uniformly dispersed. When the doping amounts are appropriate, the improvement in thermal conductivity may be attributed to two reasons: (1) the MWCNTs can be uniformly dispersed, as the SEM shows; (2) tiny thermally conductive channels may be formed on the interface between the molten salt and the MWCNTs, thereby generating a boundary effect. This kind of composite material may help improve solar heat storage and heat transfer capacity, and thereby increase the efficiency of solar thermal power generation.

rsc.li/rsc-advances

\section{Introduction}

With the gradual increase in resource consumption, energy demand is also increasing. Solar energy is an inexhaustible supply of clean energy. The use of molten salt for solar thermal power generation is one of the best ways to solve the energy problem. In a solar thermal power generation system, an effective heat transfer and heat storage system can ensure the continuous operation of the solar thermal power generation system and realize the stable utilization of solar energy. Molten salt has attracted wide attention in the industry as a promising heat transfer and heat storage medium. $60 \% \mathrm{NaNO}_{3}, 40 \% \mathrm{KNO}_{3}$ (solar salt) and $53 \% \mathrm{KNO}_{3}, 40 \% \mathrm{NaNO}_{2}, 7 \% \mathrm{NaNO}_{3}$ (Hitec) have become the two most commonly used molten salt media in CSP

${ }^{a}$ Key Laboratory of Comprehensive and Highly Efficient Utilization of Salt Lake Resources, Qinghai Institute of Salt Lakes, Chinese Academy of Sciences, Xining, 810000, China.E-mail: lijinli18@163.com; marliy001@163.com

${ }^{b}$ Key Laboratory of Salt Lake Resources Chemistry of Qinghai Province, Xining, 810000, China

'University of Chinese Academy of Sciences, Beijing, 100000, China
(Concentrating Solar Power) plants., ${ }^{\mathbf{1 , 2}}$ Compared with carbonate, ${ }^{3,4}$ chloride salt, ${ }^{5,6}$ fluoride salt, ${ }^{7,8}$ sulfate $^{9}$ or molten hydroxide, ${ }^{10}$ nitrate ${ }^{11-14}$ is the best heat storage and heat transfer medium with low viscosity, low saturated vapor pressure, low corrosivity, large specific heat and so on. However, the thermal conductivity of solar salt is only about $0.5 \mathrm{~W}(\mathrm{~m} \mathrm{~K})^{-1},{ }^{15}$ which may result in lower energy conversion efficiency and limit the utilization efficiency of solar energy. Therefore, it is of farreaching significance and practical value to construct a type of molten salt system with high thermal conductivity to improve the utilization rate of solar thermal power.

In recent decades, nanomaterials and carbon materials have been widely used in thermal energy storage and heat transfer by improving the thermal properties of materials. Zeinab Hajjar et al. added graphene oxide to deionized water and found that the thermal conductivity of the nanofluid increased significantly with the amount of graphene oxide added. ${ }^{16}$ Manila Chieruzzi et al. doped $\mathrm{Al}_{2} \mathrm{O}_{3}, \mathrm{SiO}_{2}$ or $\mathrm{TiO}_{2}$ into solar salt and found that after doping with a certain amount of nanoparticles, the temperature range of the materials was wider and the 


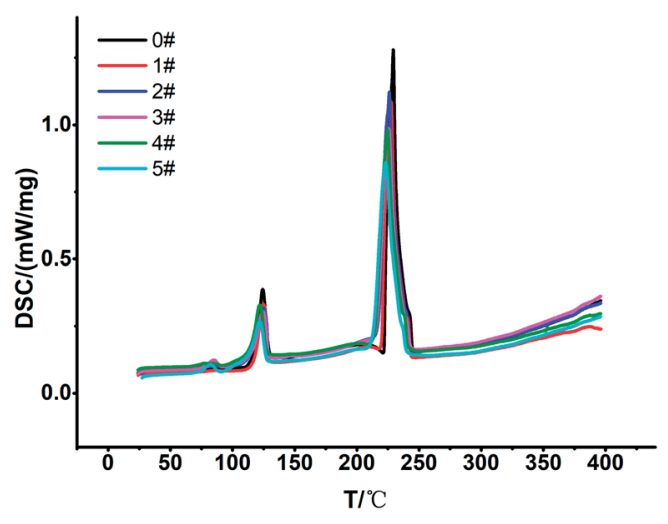

Fig. 1 DSC thermogram of solar salt doped with different doping amounts.

specific heat was greatly increased. ${ }^{17}$ They speculated that the enhancement in the thermal effect was due to the interface effect caused by the high specific surface area and the high specific surface energy of the nanoparticles. Donghyun Shin et al. mixed potassium carbonate and lithium carbonate with nano- $\mathrm{Al}_{2} \mathrm{O}_{3}$ to obtain a kind of salt-based nanofluid and found that the specific heat increased significantly. ${ }^{18}$ They speculated that the chain-like nanostructures formed in the salt-based nanofluids acted to increase the specific heat of the material. Qiangzhi Xie et al. doped graphene nanosheets into solar salt, and found that adding $1 \%$ graphene nanosheets can the increase specific heat by $16.7 \% .^{19}$ They found that adding more nanoparticles was not better and speculated that with a further increase in the concentration of nanoparticles, the nano-effects gradually weakened. Zoubir Acem et al., doping expanded graphite into a mixed molten salt of $\mathrm{KNO}_{3}$ and $\mathrm{NaNO}_{3}$, found that the phase transition temperature of the material was almost unchanged, but the thermal conductivity increased by about 20 times. $^{20,21}$ And by simulating an application model with a computer, they estimated that using such graphite/salt composites can significantly reduce the investment cost of the storage system. Majid TabkhPaz et al. used a computer to simulate the composite material obtained by mixing carbon nanotubes and hexagonal boron nitride. ${ }^{22}$ It was predicted that the addition of carbon nanotubes could greatly improve the thermal conductivity of the material and this was confirmed experimentally. Geng Qiao et al. carried out molecular dynamics simulations of molten salt with nitric acid doped silica nanoparticles, indicating that a layer of molecules adhered to the surface of the nanoparticles, and the specific heat of the thermal conductivity of the nitrate greatly increased. ${ }^{23}$

Since the discovery of CNTs in $1991,{ }^{24}$ research on CNTs has developed rapidly. Due to their unique nanoscale size structure, carbon nanomaterials have a special nanometer effect. ${ }^{25}$ Compared with traditional materials, carbon nanotubes, as unique one-dimensional nano-materials, have the advantages of good mechanical stability, high strength and excellent thermal conductivity. ${ }^{26-29}$ Multi-walled carbon nanotubes with a thermal conductivity of up to $3000 \mathrm{~W}(\mathrm{~m} \mathrm{~K})^{-1}$, are ultra-high thermal conductivity materials. ${ }^{30}$ The use of their high thermal conductivity characteristics, composing them with traditional materials, can greatly improve the thermal performance of these traditional materials, and obtain high thermal conductivity for the composite material. Esraa Hamdy et al. prepared solar salt/MWCNTs composites using an ultrasonic oscillation method and found that the thermal conductivity was improved, and the specific heat was also increased at certain doping amounts. ${ }^{31}$

In the present study, due to the excellent thermal conductivity of MWCNTs, solar salt was mixed with MWCNTs by the high-temperature melting method to obtain a composite material with high thermal conductivity. We controlled the doping amounts over a smaller range and used a preparation method different from that of Esraa Hamdy et al. Different preparation processes produced some differences in the material's thermal properties. Then the structure-activity relationship of various thermal properties of the material was analyzed. The effects of doped MWCNTs on the thermal conductivity of the material were further discussed by means of microscopic characterization.

\section{Experimental section}

\subsection{Materials and sample preparation}

Six sets of different ratios of sodium nitrate (purity: >99 wt\%), potassium nitrate (purity: $>99 \mathrm{wt} \%$ ) and multi-walled carbon nanotubes (purity: >95 wt\%; diameter: $12-15 \mathrm{~nm}$; length: $3-15$ $\mu \mathrm{m}$; China Nanjing Xianfeng Nano Material Technology Co., Ltd.) were weighed so that the mass ratio of sodium nitrate to potassium nitrate in these six samples was $6: 4$ and the amounts of multiwalled carbon nanotubes were $0 \%, 0.1 \%$, $0.2 \%, 0.3 \%, 0.4 \%, 0.5 \%$ (the samples were numbered $0-5 \#$ ). The six samples were placed in corundum crucibles, stirred and mixed thoroughly. The crucibles were placed in a Muffle furnace and heated at $400{ }^{\circ} \mathrm{C}$ for $4 \mathrm{~h}$. Then they were removed onto a ceramic plate and cooled to $300{ }^{\circ} \mathrm{C}$, grounded to powder after cooling to room temperature, then sealed in dry bags to keep.

\subsection{Thermophysical characterization}

The melting point, phase transition enthalpy and decomposition temperature of these 6 samples were measured by simultaneous thermogravimetry - differential scanning calorimeter (TG-DSC, US Thermo Electric Company, SDT Q600). The tests were carried out in nitrogen over a temperature range of 35$700{ }^{\circ} \mathrm{C}$ with a heating rate of $5{ }^{\circ} \mathrm{C} \mathrm{min}^{-1}$.

The density of these 6 samples was tested with a hightemperature molten salt density meter (Shanghai Institute of Applied Physics, Chinese Academy of Sciences) by the Archimedes method. The accuracy was within $\pm 1 \%$.

The specific heat of these 6 samples was tested by a differential scanning calorimeter (NETZSCH DSC200F3). The threestep test method followed standard ASTM E1269. We had measured the DSC baseline values for the empty crucible, the DSC reference values for standard sapphire and the DSC values for the samples. Then the specific heat of the samples can be calculated based on the three DSC values. The accuracy 

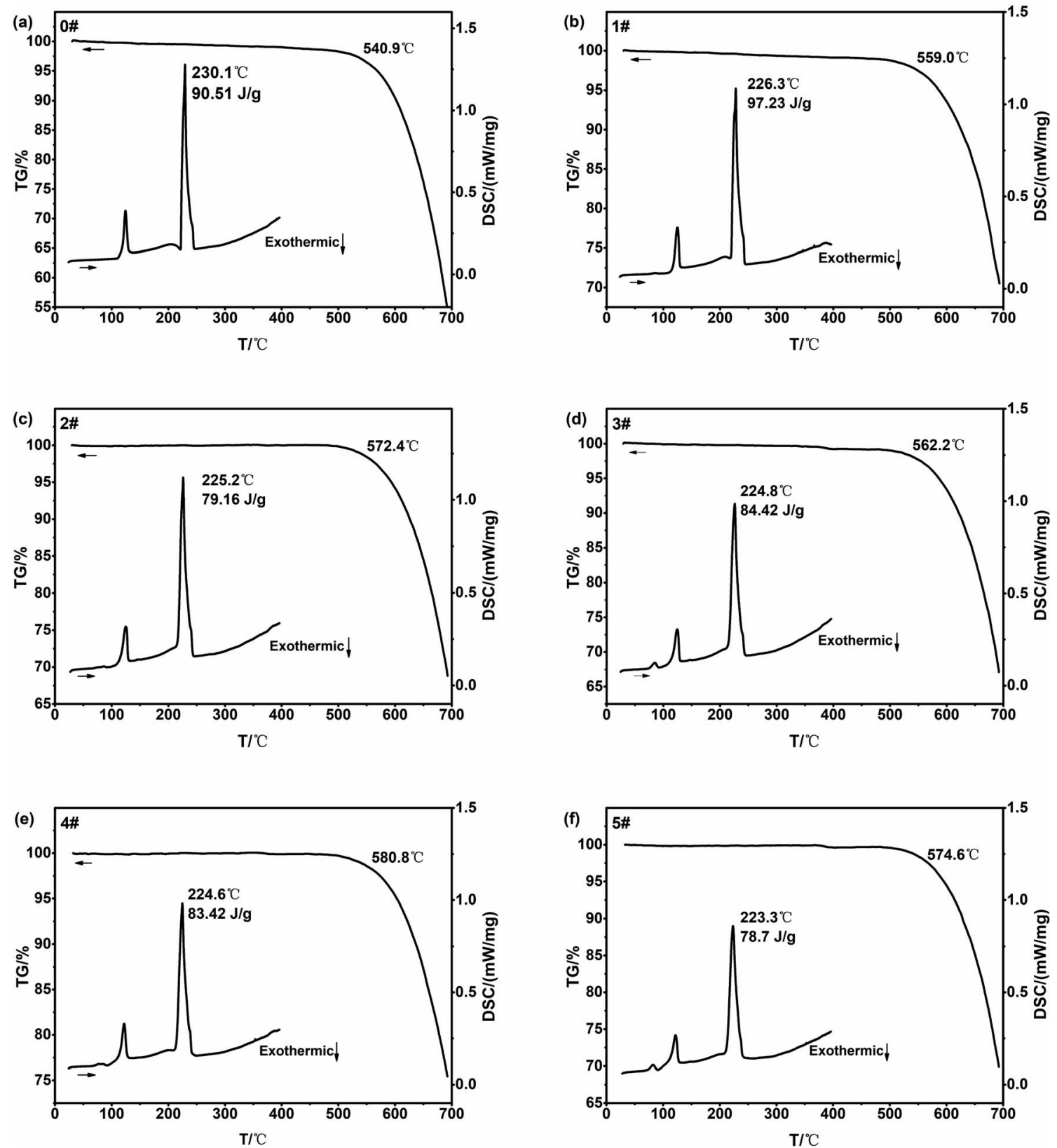

Fig. 2 TG-DSC patterns of (a) solar salt; (b) solar salt doped with $0.1 \%$ MWCNTs; (c) solar salt doped with $0.2 \%$ MWCNTs; (d) solar salt doped with $0.3 \%$ MWCNTs; (e) solar salt doped with 0.4\% MWCNTs; (f) solar salt doped with $0.5 \%$ MWCNTs.

was within $\pm 2 \%$. The temperature range was from $30{ }^{\circ} \mathrm{C}$ to $400{ }^{\circ} \mathrm{C}$, and the heating rate was $5{ }^{\circ} \mathrm{C} \mathrm{min}^{-1}$.

These 6 samples were tested for thermal diffusivity using a laser thermal conductivity meter (Linseis LFA1000). The test was performed in helium and the thermal conductivity was calculated from the density and specific heat data.

\subsection{Microscopic characterization}

XRD (X'pert Pro) was used to analyze the changes in the composite materials.

X-ray photoelectron spectroscopy (ESCALAB 250Xi, XPS) was used to analyze the $\mathrm{C}$ 1s spectra of two samples (5\#) before and after melting, and the changes in the binding energies of the $\mathrm{C}$ element before and after melting were compared. 


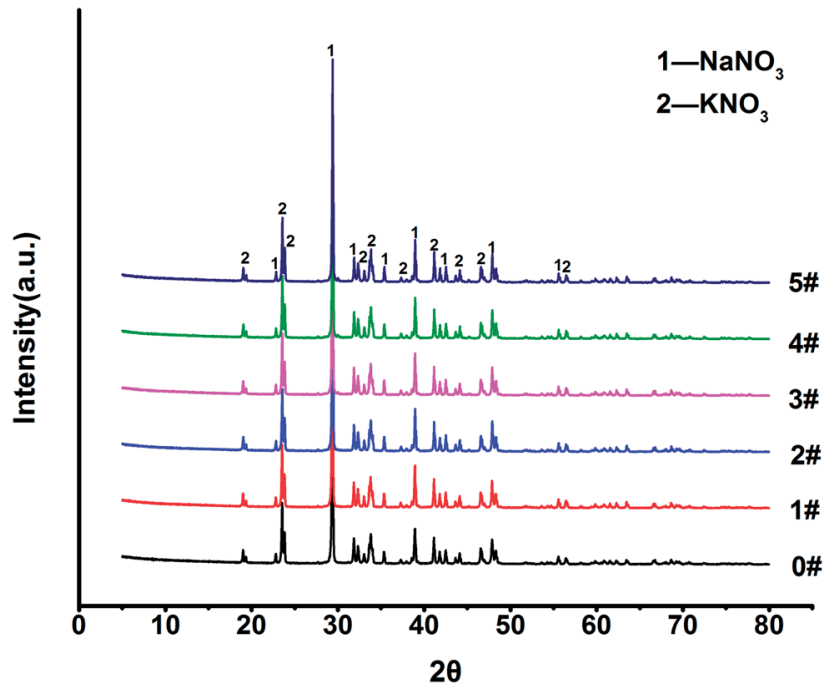

Fig. 3 XRD patterns of solar salt doped with different doping amounts.

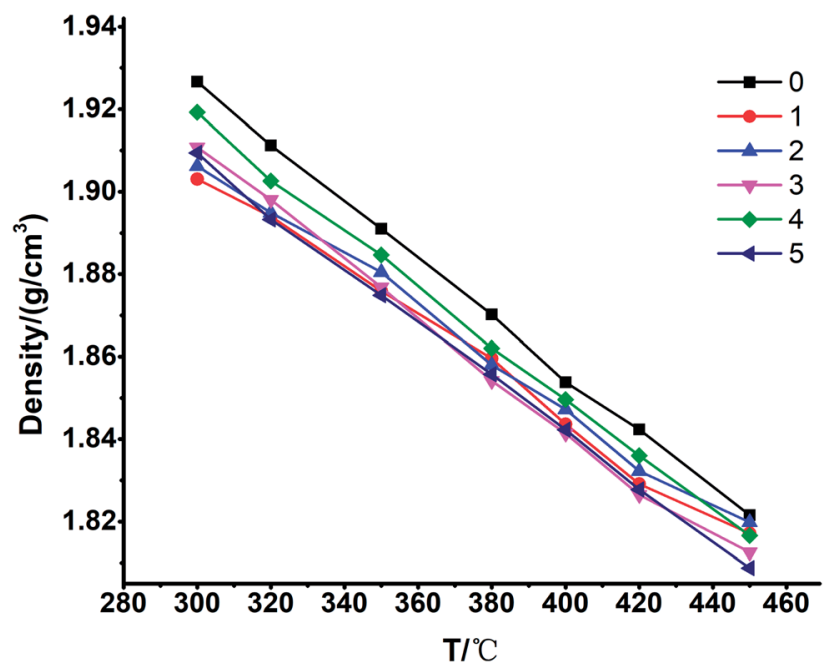

Fig. 4 Density of solar salt doped with different doping amounts.

Table 1 The fitting equation of density with temperature

\begin{tabular}{llll}
\hline & \multicolumn{2}{l}{$\rho(T)=a+b T\left(\rho: \mathrm{g} \mathrm{cm}^{-3}\right)$} & \\
\cline { 2 - 3 } Samples & $a$ & $b$ & $R$-square \\
\hline $0 \#$ & 2.13942 & $-7.10148 \times 10^{-4}$ & 0.9986 \\
$1 \#$ & 2.07995 & $-5.84356 \times 10^{-4}$ & 0.9873 \\
$2 \#$ & 2.08230 & $-5.90192 \times 10^{-4}$ & 0.9942 \\
$3 \#$ & 2.12164 & $-7.00705 \times 10^{-4}$ & 0.9990 \\
$4 \#$ & 2.12415 & $-6.85046 \times 10^{-4}$ & 0.9980 \\
$5 \#$ & 2.10689 & $-6.63511 \times 10^{-4}$ & 0.9987 \\
\hline
\end{tabular}

The microstructures of these samples were analyzed by a Scanning Electron Microscope (NanoSEM400) and a Transmission Electron Microscope (FEI-TECNAI G2 TF20).

The Raman spectra were analyzed at $25^{\circ} \mathrm{C}$ and $300{ }^{\circ} \mathrm{C}$ by a Micromanifold Raman spectrometer (Thermo Scientific ${ }^{\mathrm{TM}}$ DXR).

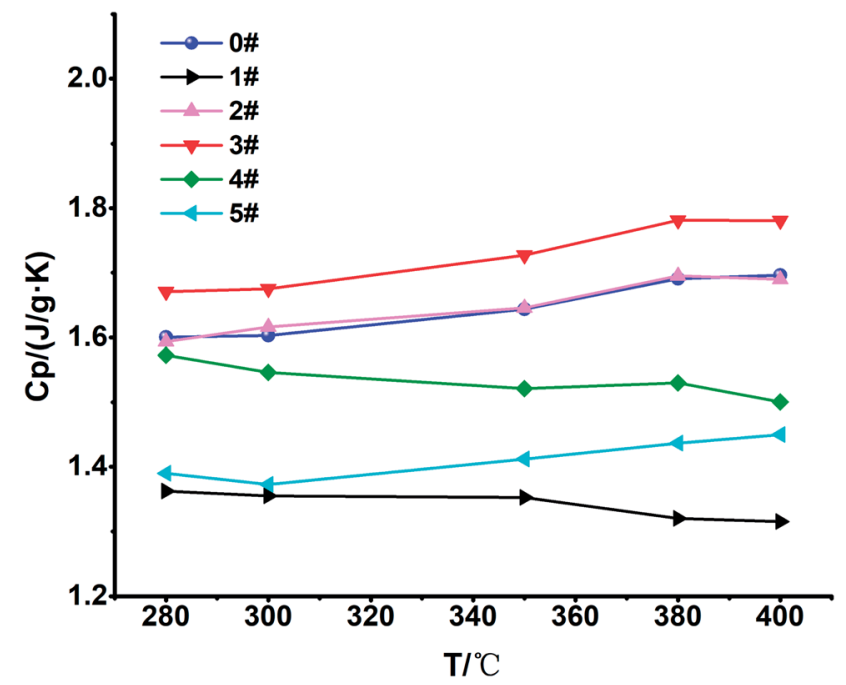

Fig. 5 Specific heat of solar salt doped with different doping amounts.

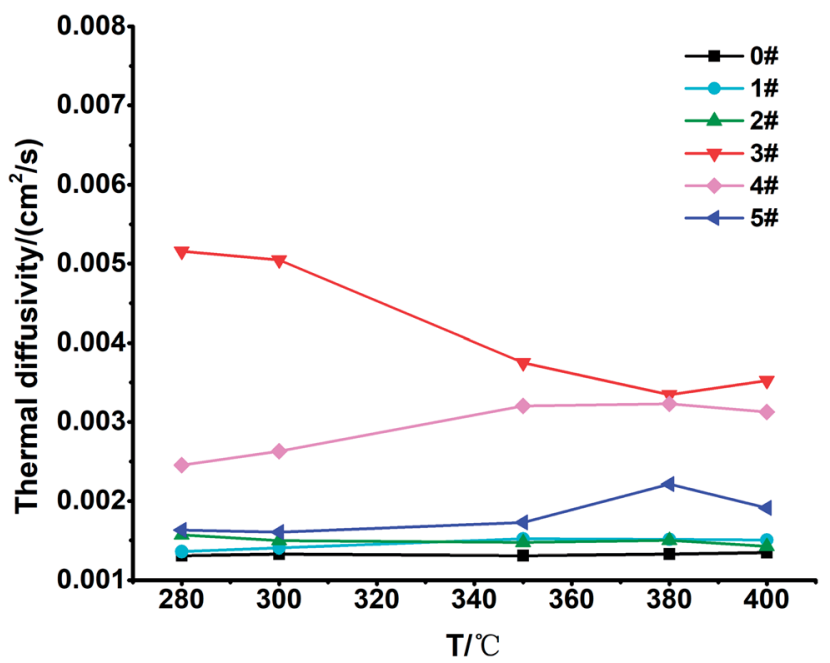

Fig. 6 Thermal diffusivity of solar salt doped with different doping amounts.

\section{Results and discussion}

\subsection{TG-DSC, XRD, density}

3.1.1 Melting point, enthalpy of phase transition and decomposition temperature. The DSC thermogram is shown in Fig. 1 and the melting point, enthalpy of phase transition and decomposition temperature are shown in Fig. 2 (0\#-5\#). We can see that after doping with $0.1-0.5 \%$ multi-walled carbon nanotubes, respectively, the melting points of these five samples reduced by $3.8-6.8{ }^{\circ} \mathrm{C}$ and the decomposition temperature increased by $21.3-39.9^{\circ} \mathrm{C}$ compared with the blank sample $0 \#$. (The decomposition temperature value was taken at a mass fraction of $97 \%$ ). In general, the melting point and decomposition temperature of the composite materials changed very little, and the application temperature range of the composite materials was slightly larger than for solar salt. 


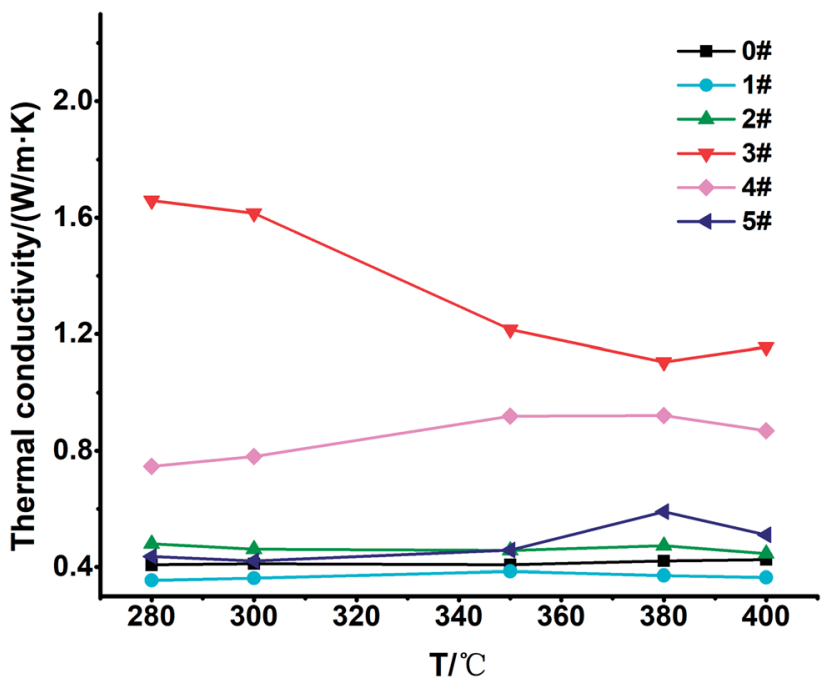

Fig. 7 Thermal conductivity of solar salt doped with different doping amounts.
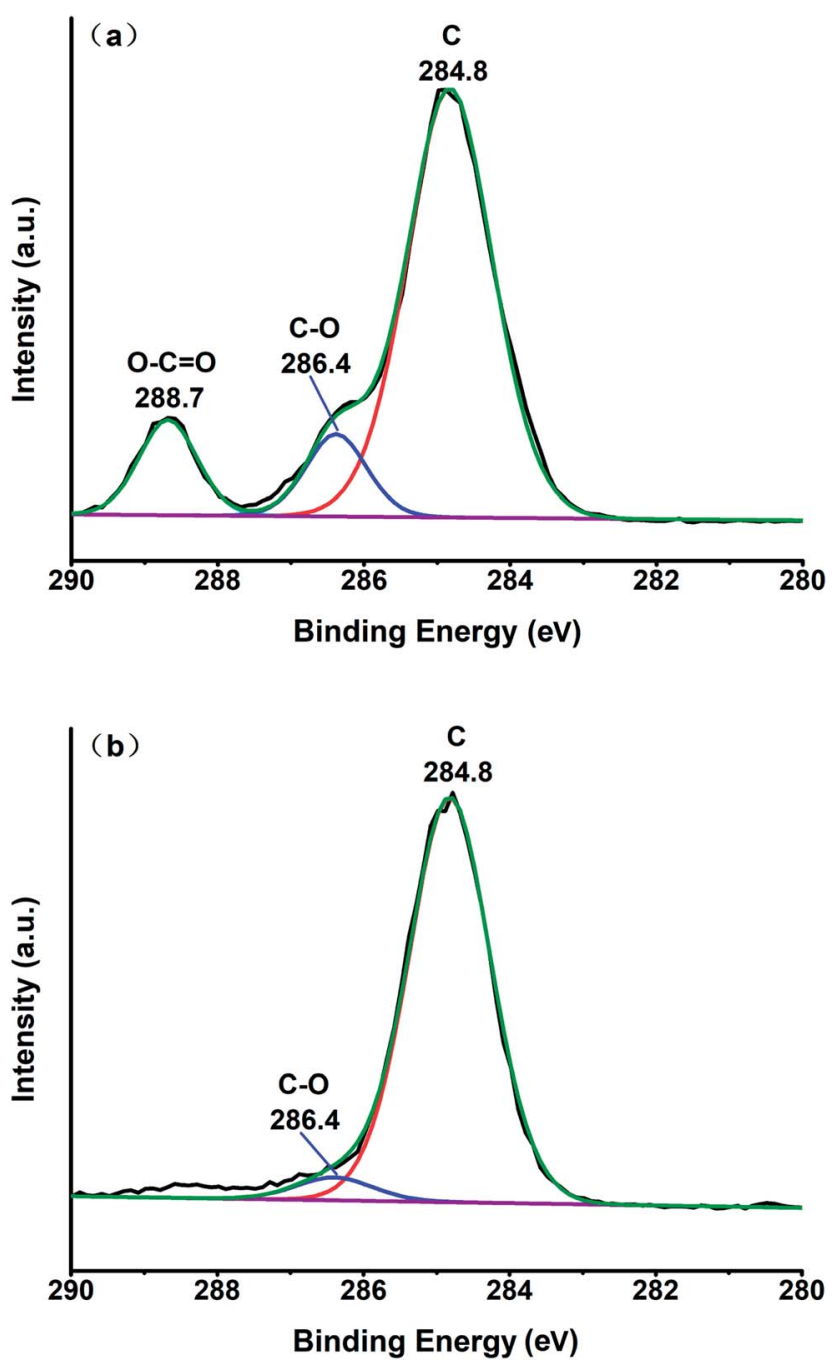

Fig. 8 (a) XPS spectra of sample 5\# before melting; (b) XPS spectra of sample $5 \#$ after melting.

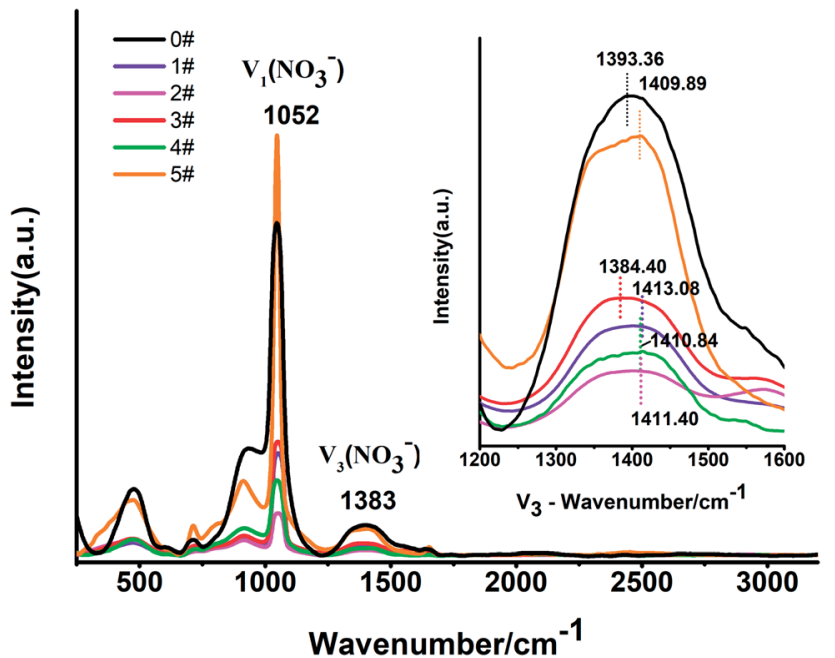

Fig. 9 Raman spectroscopy of solar salt doped with different doping amounts at $300^{\circ} \mathrm{C}$.

3.1.2 Component analysis. The XRD patterns of these six samples are given in Fig. 3, which shows that the sample components are sodium nitrate and potassium nitrate, while the doped multi-walled carbon nanotubes do not change the composition of the composite salt. The doping amounts of MWCNTs were too small, so the main body of the material was still $\mathrm{NaNO}_{3} / \mathrm{KNO}_{3}$ with a mass ratio $6: 4$, and the melting point and decomposition temperature were also almost unchanged.

3.1.3 Density. The density was tested by the Archimedes method and the data are shown in Fig. 4. We can see the linear relationship between density and temperature. The fitting equation is shown in Table 1. After doping with different amounts of multi-walled carbon nanotubes, the density of these samples decreased slightly compared with the non-doped blank samples.

\subsection{Specific heat}

The specific heat test results of these 6 samples are shown in Fig. 5. We found that the specific heat of the blank sample $0 \#$ is $1.6029 \mathrm{~J} \mathrm{~g}^{-1} \mathrm{~K}^{-1}$ at $300{ }^{\circ} \mathrm{C}$, and the specific heat of sample $3 \#$ was increased by $4.50 \%$, reaching $1.67505 \mathrm{~J} \mathrm{~g}^{-1} \mathrm{~K}^{-1}$. The specific heat of sample $2 \#$ was slightly increased by $0.81 \%$, reaching $1.61594 \mathrm{~J} \mathrm{~g}^{-1} \mathrm{~K}^{-1}$, and the other samples had a lower specific heat.

\subsection{Thermal diffusivity and thermal conductivity}

The thermal diffusivity of the 6 samples was tested using a laser thermal conductivity meter. The data for each temperature point were averaged after five measurements. The data results are shown in Fig. 6 . We found that the thermal diffusivity of the blank sample $0 \#$ was $0.00133 \mathrm{~cm}^{2} \mathrm{~s}^{-1}$ at $300{ }^{\circ} \mathrm{C}$, and the thermal diffusivity of sample $3 \#$ increased the most, by $280 \%$ to 0.00505 $\mathrm{cm}^{2} \mathrm{~s}^{-1}$. The thermal diffusivity of sample $4 \#$ increased by $98 \%$ to $0.00263 \mathrm{~cm}^{2} \mathrm{~s}^{-1}$, and the thermal diffusivity of the other samples increased slightly. 

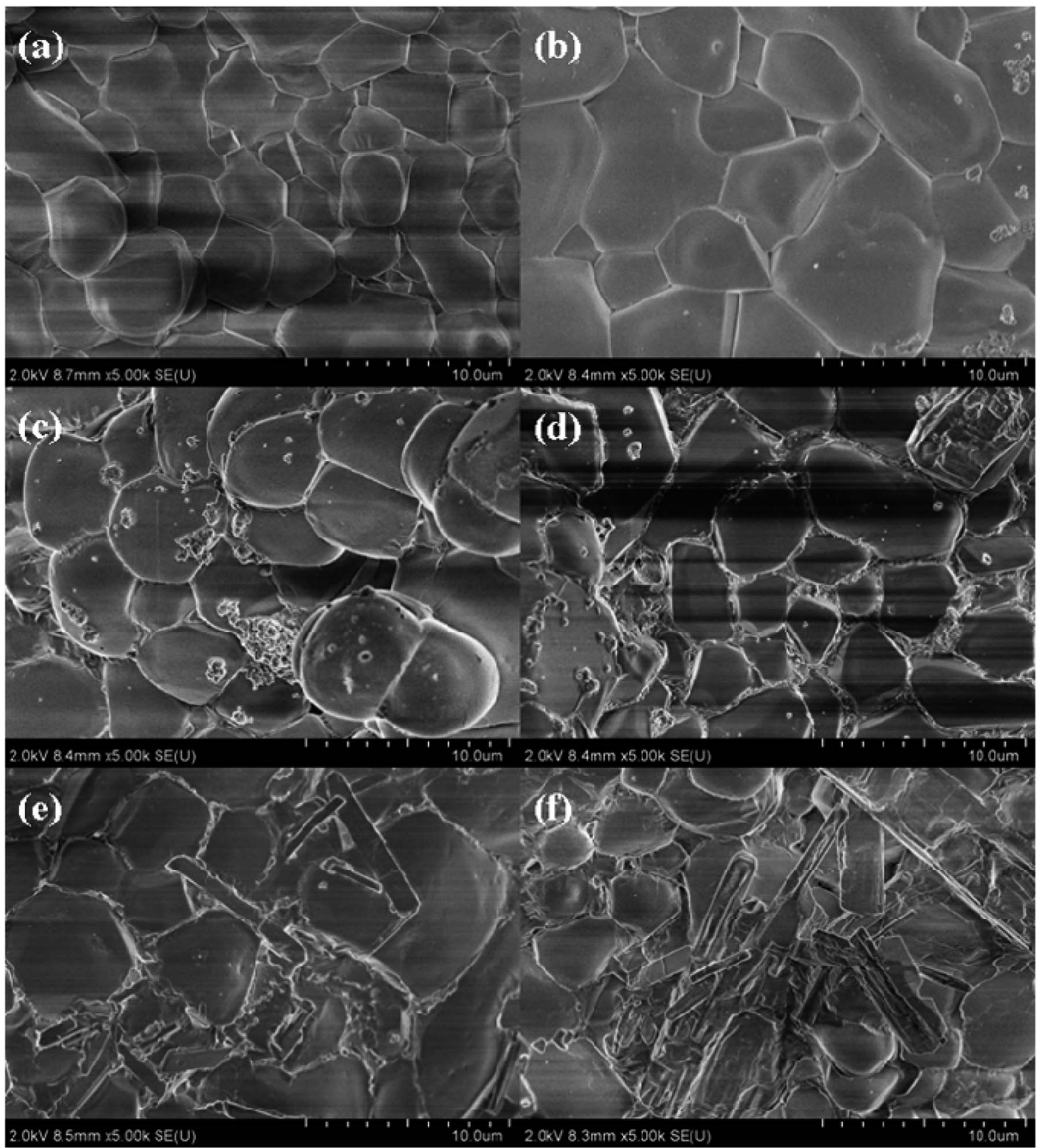

Fig. 10 SEM images at $10.0 \mu \mathrm{m}$ of (a) solar salt; (b) solar salt doped with $0.1 \%$ MWCNTs; (c) solar salt doped with $0.2 \%$ MWCNTs; (d) solar salt doped with 0.3\% MWCNTs; (e) solar salt doped with 0.4\% MWCNTs; (f) solar salt doped with 0.5\% MWCNTs.

According to the calculation formula for thermal conductivity: $k=\alpha \rho C_{\mathrm{p}}\left(\alpha\right.$ : thermal diffusivity; $\rho$ : density; $C_{\mathrm{p}}$ : specific heat), the thermal conductivity of the 6 groups of samples was calculated, and the results are shown in Fig. 7.

We found that the thermal conductivity of the blank sample 0\# was $0.41073 \mathrm{~W}(\mathrm{~m} \mathrm{~K})^{-1}$ at $300{ }^{\circ} \mathrm{C}$, and the thermal conductivity of sample $3 \#$ increased the most, by $293 \%$, reaching $1.61534 \mathrm{~W}\left(\mathrm{~m} \mathrm{~K}^{-1}\right.$; the thermal conductivity of sample $4 \#$ increased by $90 \%$ to $0.77987 \mathrm{~W}(\mathrm{~m} \mathrm{~K})^{-1}$. The thermal conductivity of sample $2 \#$ and sample $5 \#$ increased slightly and the thermal conductivity of sample $1 \#$ decreased slightly.

\subsection{XPS}

Two samples 5\# (before and after melting) were analyzed by XPS spectroscopy, and the resultant fitting of the $\mathrm{C} 1 \mathrm{~s}$ peak is shown in Fig. 8. As can be seen from Fig. 8(a), carbon, hydroxyl (C-O) and carboxyl $(\mathrm{O}-\mathrm{C}=\mathrm{O})$ were present at $284.8 \mathrm{eV}, 286.4 \mathrm{eV}$ and $288.7 \mathrm{eV}$, respectively, in the sample before melting. ${ }^{32}$ These functional groups come from the original multi-walled carbon nanotubes. We can see from Fig. 8(b) that the carbon and hydroxyl functional groups (C-O) were present only at $284.8 \mathrm{eV}$ and $286.4 \mathrm{eV}$ in the sample after melting, and the number of hydroxyl groups $(\mathrm{C}-\mathrm{O})$ was greatly reduced. We speculated that 


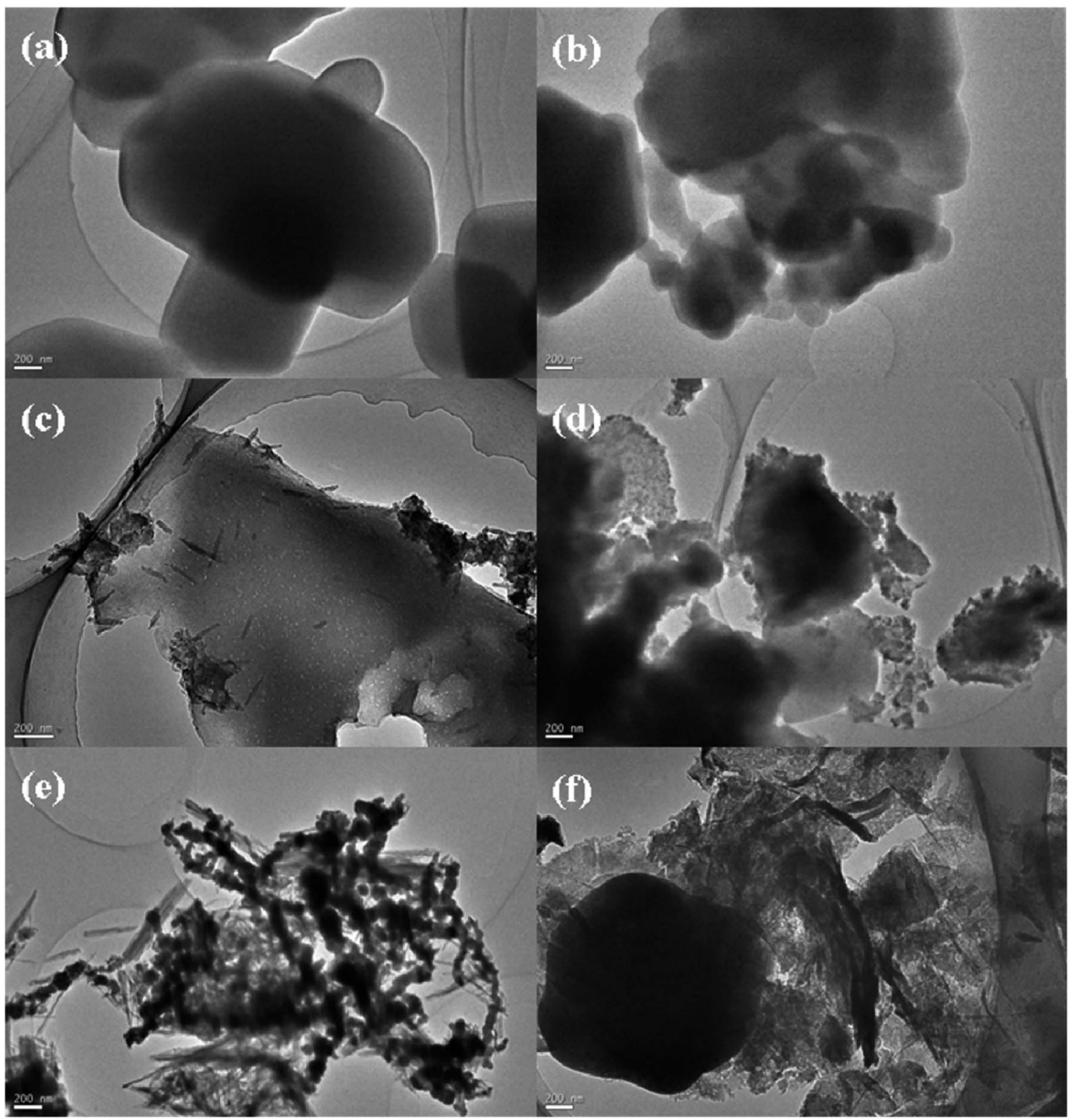

Fig. 11 TEM images at $200 \mathrm{~nm}$ of (a) solar salt; (b) solar salt doped with $0.1 \%$ MWCNTs; (c) solar salt doped with $0.2 \%$ MWCNTs; (d) solar salt doped with $0.3 \%$ MWCNTs; (e) solar salt doped with $0.4 \%$ MWCNTs; (f) solar salt doped with $0.5 \%$ MWCNTs.

the MWCNTs were purified and the impurities were removed due to the interaction of the multi-walled carbon nanotubes and nitrate at high temperature.

\subsection{Raman spectroscopy}

The Raman spectra of the 6 samples were tested at $300^{\circ} \mathrm{C}$. From the shift in the $\mathrm{V}_{3}\left(\mathrm{NO}_{3}{ }^{-}\right)$peak we find that the shift of the blank sample $0 \#$ was $1393.36 \mathrm{~cm}^{-1}{ }^{33}$ The $\mathrm{V}_{3}$ peak of sample $3 \#$ redshifted to $1384.40 \mathrm{~cm}^{-1}$, while the $\mathrm{V}_{3}$ peaks of the other samples blue-shifted. Among these, sample 1\# had the most blue-shifted, and sample $5 \#$ had the least blue-shifted. The researches of Jianfeng Xu and Sean Kelly et al. showed that the Raman peaks tended to blue-shift with a decrease in crystal size. ${ }^{34,35}$ And Janz G. J. et al. believed that the Raman shift of
$\mathrm{NO}_{3}{ }^{-}$was related to the intensity of the $\mathrm{N}-\mathrm{O}$ bond. ${ }^{36} \mathrm{We}$ speculated that the particle size of the samples became smaller and a blue-shift occurred when the MWCNTs were doped. Meanwhile, with an increase in MWCNTs content, the polarization of $\mathrm{Na}^{+} / \mathrm{K}^{+}$was weakened, the density of the $\mathrm{N}-\mathrm{O}$ electron cloud in $\mathrm{NO}^{3-}$ decreased, and the $\mathrm{N}-\mathrm{O}$ bond strength decreased, making the $\mathrm{V}_{3}$ peak red-shift. When the red-shift and the blue-shift occurred at the same time, the blue-shift decreased as the doping amounts of MWCNTs increased, and when a certain doping amount was reached, the blue-shift changed to redshift, after which a gradually decreasing blue-shift occurred again. Compared to the other samples, only sample $3 \#$ had a red-shift. We thought that the red shift in the $3 \#$ sample played the dominant role. When the addition amount was $0.3 \%$, the dispersion of MWCNTs in the molten salt may be the most 


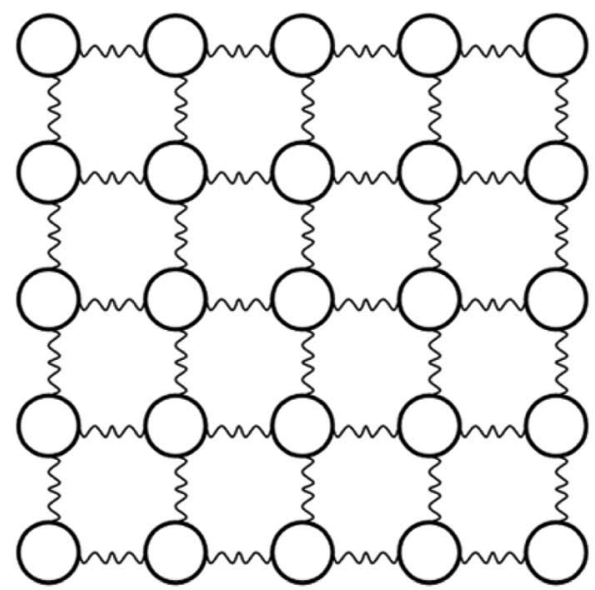

(a)

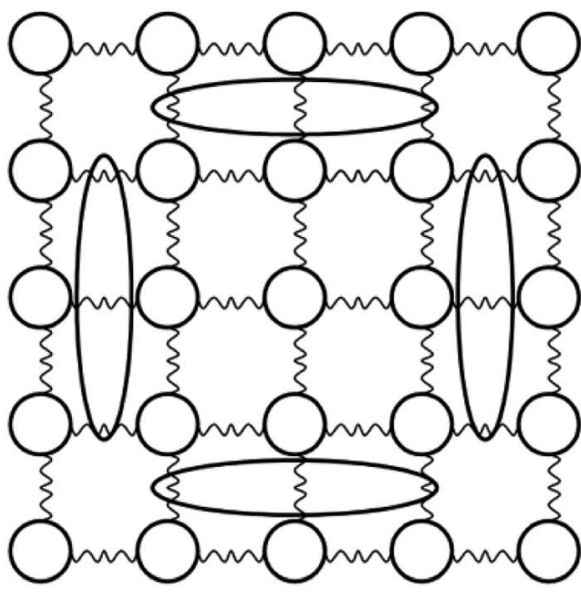

(b)

Fig. 12 (a) A schematic of nitrate in the ideal molten state (circles represent $\mathrm{Na}^{+}, \mathrm{K}^{+}$and $\mathrm{NO}_{3}{ }^{-}$); (b) a schematic of nitrate doped with $\mathrm{MWCNTs}$ in the ideal molten state (ovals represent the MWCNTs).

uniform, and the strength of the $\mathrm{N}-\mathrm{O}$ bond was reduced most (Fig. 9).

\subsection{Micromorphology analysis}

These 6 samples were observed by SEM, and the results at $10 \mu \mathrm{m}$ are shown in Fig. 10. We found that the amount of doping in sample $1 \#$ was too little and almost no carbon nanotubes existed in the particle gaps. There existed a bit of MWCNTs filling in the particle gaps of sample $2 \#$, but the dispersion was uneven. In sample $4 \#$ and sample $5 \#$, long strips of carbon nanotubes were observed due to the amounts of doping being so large that the carbon nanotubes probably reunited. Only in sample 3\# were the carbon nanotubes uniformly dispersed in the gaps between the inorganic salt particles. These 6 samples were further observed by TEM, and the results at $200 \mathrm{~nm}$ are shown in Fig. 11. From the TEM images we found that the MWCNTs were cut into short tubes under the oxidation of nitrates at high temperatures. In sample $1 \#$, the amount of MWCNTs doped was too little to be observed. The MWCNTs in sample $4 \#$ and sample $5 \#$ were agglomerated and longer than those in sample 2\#. We thought that a larger aspect ratio would not contribute to an improvement in thermal performance. There were hardly any visible MWCNTs in sample 3\#, probably because the MWCNTs were cut too short and dispersed so well that they were dispersed in the inorganic salt gap and were not observed on the surface.

Donghyun Shin et al. proposed the idea that there was a semi-solid layer between the nanoparticles and the fluid, and that these semi-solid layers generally had higher thermal properties than conventional liquids. ${ }^{37}$ We speculated that when the materials were doped with MWCNTs, tiny thermally conductive channels with high thermal conductivity may be formed on the interface between the molten salt and the MWCNTs, and that the influence of the interface thermal resistance was overcome to some extent.

\subsection{Mechanism model}

Through the thermophysical characterization, we found that doping with MWCNTs can improve the thermal properties of solar salt. In the range of $0.1-0.5 \%$ doping, the thermal conductivity increased first and then decreased, and the thermal conductivity of sample $3 \#$ improved the most. By Raman and SEM/TEM characterization, we speculated that the MWCNTs were uniformly dispersed in the gaps between the inorganic salt particles in sample $3 \#$, which also led to the greatest reduction in $\mathrm{N}-\mathrm{O}$ bond strength. And tiny channels with high thermal conductivity may be formed on the interface between the molten salt and the MWCNTs, thereby generating a boundary effect. A schematic of nitrate in the ideal molten state may be as shown in Fig. 12(a), where heat is conducted in ionic liquids from nitrate to nitrate. The ideal distribution schematic of nanoparticles and molten salt in sample $3 \#$ may be as shown in Fig. 12(b), where heat is conducted from ionic liquids to the MWCNTs and then from the MWCNTs to ionic liquids. When the doping amount was $0.3 \%$, the dispersibility and the boundary effect may be the best, so that its thermal performance was the most improved.

\section{Conclusions}

In this study, the thermal properties of solar salt doped with multi-walled carbon nanotubes have been improved, which may have some application advantages in solar thermal power generation. The results showed that when doped with multiwalled carbon nanotubes, compared with pure solar salt, the melting point of the composite material slightly decreased, the decomposition temperature slightly increased, and the density slightly decreased. Especially when doped with $0.3 \%$ MWCNTs, the specific heat and thermal diffusivity increased, and the thermal conductivity increased by $293 \%$. Analysis of the results of XPS showed that the MWCNTs had been purified and a large number of impurity groups had been removed from the 
samples after the high-temperature melting. By analyzing the Raman spectra, we speculated that the presence of MWCNTs weakened the polarization of $\mathrm{Na}^{+} / \mathrm{K}^{+}$and weakened the strength of the $\mathrm{N}-\mathrm{O}$ bond. And according to the SEM and TEM observations, when the doping amount was $0.3 \%$, the dispersion of MWCNTs was the best. The use of this new type of composite material was conducive to improving the heat transfer efficiency of molten salt in thermoelectric power generation and opening up new ways for solar thermal utilization.

\section{Conflicts of interest}

There are no conflicts of interest to declare.

\section{Acknowledgements}

This work was supported by the funding of Science and Technology Service Network Initiative (KFJ-EW-STS-131), Major Scientific and Technological Special Project of Qinghai Province (2017-GX-A3) and the National Natural Science Fund projects of China (No. 21601197).

\section{References}

1 T. Bauer, D. Laing and R. Tamme, Recent Progress in Alkali Nitrate/Nitrite Developments for solar Thermal Power Applications, Molten Salts Chemistry and Technology, MS9, June 5-9, 2011, Trondheim, Norway, 2011, pp. 1-10.

2 J. Lopez, Z. Acem and E. P. Del Barrio, $\mathrm{KNO}_{3} / \mathrm{NaNO}_{3}$-Graphite materials for thermal energy storage at high temperature: part II. - phase transition properties, Appl. Therm. Eng., 2010, 30, 1586-1593.

3 B. C. Shin, D. K. Sang and W. H. Park, Ternary carbonate eutectic (lithium, sodium and potassium carbonates) for latent heat storage medium, Sol. Energy Mater., 1990, 21(1), 81-90.

4 N. Araki, M. Matsuura, A. Makino, et al., Measurement of thermophysical properties of molten salts: Mixtures of alkaline carbonate salts, Int. J. Thermophys., 1988, 9(6), 1071-1080.

5 X. Wei, M. Song, W. Wang, et al., Design and thermal properties of a novel ternary chloride eutectics for hightemperature solar energy storage, Appl. Energy, 2015, 156(1), 306-310.

6 P. D. Myers Jr and D. Y. Goswami, Thermal energy storage using chloride salts and their eutectics, Appl. Therm. Eng., 2016, 109, 889-900.

7 Y. M. Xing, X. Xu, X. G. Yuan, et al., Numerical simulation of high-temperature phase change heat storage system, Heat Transfer-Asian Research, 2002, 33(1), 32-41.

8 D. L. Jacobson and R. Ponnappan, Performance of a cylindrical phase-change thermal energy storage unit, AIAA J., 1982, 21(5), 774-780.

9 B. M. Suleiman, M. Gustavsson, E. Karawacki, et al., Thermal properties of lithium sulphate, J. Phys. D: Appl. Phys., 1997, 30(18), 2553.
10 R. T. Coyle, T. M. Thomas and G. Y. Lai, Exploratory corrosion tests on alloys in molten salts at $900{ }^{\circ} \mathrm{C}, \mathrm{J}$. Mater. Energy Syst., 1986, 7(4), 345-352.

11 W. D. Steinmann and R. Tamme, Latent Heat Storage for Solar Steam Systems, J. Mater. Energy Syst., 2008, 130(02), 011004.

12 Z. Yang and S. V. Garimella, Thermal analysis of solar thermal energy storage in a molten-salt thermocline, Sol. Energy, 2010, 84(6), 974-985.

13 D. Laing, T. Bauer and W. D. Steinmann, et al., Advanced high temperature latent heat storage system - design and test results// Effstock, 2009.

14 D. Kearney, U. Herrmann, P. Nava, et al., Assessment of a Molten Salt Heat Transfer Fluid in a Parabolic Trough Solar Field, J. Mater. Energy Syst., 2002, 125(2), 293-299.

15 R. Serrano-López, J. Fradera and S. Cuesta-López, Molten salts database for energy applications, Chem. Eng. Process., 2013, 73(73), 87-102.

16 Z. Hajjar, A. M. Rashidi and A. Ghozatloo, Enhanced thermal conductivities of graphene oxide nanofluids, Int. Commun. Heat Mass Transfer, 2014, 57, 128-131.

17 M. Chieruzzi, G. F. Cerritelli, A. Miliozzi, et al., Effect of nanoparticles on heat capacity of nanofluids based on molten salts as PCM for thermal energy storage, Nanoscale Res. Lett., 2013, 8(1), 448.

18 D. Shin and D. Banerjee, Specific heat of nanofluids synthesized by dispersing alumina nanoparticles in alkali salt eutectic, Int. J. Heat Mass Transfer, 2014, 74(5), 210-214.

19 Q. Xie, Q. Zhu and L. Yan, Thermal Storage Properties of Molten Nitrate Salt-Based Nanofluids with Graphene Nanoplatelets, Nanoscale Res. Lett., 2016, 11(1), 1-7.

$20 \mathrm{Z}$. Acem, J. Lopez and E. P. D. Barrio, $\mathrm{KNO}_{3} / \mathrm{NaNO}_{3}-$ Graphite materials for thermal energy storage at high temperature: part I. - elaboration methods and thermal properties, Appl. Therm. Eng., 2010, 30(13), 1580-1585.

$21 \mathrm{~J}$. Lopez, Z. Acem and E. P. D. Barrio, $\mathrm{KNO}_{3} / \mathrm{NaNO}_{3}-$ Graphite materials for thermal energy storage at high temperature: part II. - phase transition properties, Appl. Therm. Eng., 2010, 30(13), 1586-1593.

22 M. Tabkhpaz, S. Shajari, M. Mahmoodi, et al., Thermal conductivity of carbon nanotube and hexagonal boron nitride polymer composites, Composites, Part B, 2016, 100, 19-30.

23 G. Qiao, A. Alexiadis and Y. Ding, Simulation study of anomalous thermal properties of molten nitrate salt, Powder Technol., 2017, 314, 660-664.

24 S. Iijima, Helical microtubules of graphitic carbon, Nature, 1991, 354(6348), 56-58.

25 V. Harik, Trends in nanoscale mechanics: Mechanics of carbon Nanotubes, Graphene, Nanocomposites and molecular dynamics, Springer Science+Business Media Dordrecht, 2014, pp. 19-44.

26 J. P. Salvetat, J. M. Bonard and R. Bacsa, et al., Physical properties of carbon nanotubes, International Winterschool on Electron, American Institute of Physics, 1998, pp. 467-480. 
27 S. Berber, Y. K. Kwon and D. Tomanek, Unusually high thermal conductivity of carbon nanotubes, High Thermal Conductivity Materials, Springer New York, 2006, p. 4613.

28 R. S. Ruoff and D. C. Lorents, Mechanical and thermal properties of carbon nanotubes, Carbon, 1995, 33(7), 925930.

29 J. C. Lasjaunias, Thermal properties of carbon nanotubes, $C$. R. Phys., 2003, 4(9), 1047-1054.

30 P. Kim, L. Shi, A. Majumdar, et al., Thermal transport measurements of individual multiwalled nanotubes, Phys. Rev. Lett., 2001, 87(21), 215502.

31 E. Hamdy, S. Ebrahim and F. Abulfotuh, et al., Effect of multi-walled carbon nanotubes on thermal properties of nitrate molten salts, Renewable and Sustainable Energy Conference, IEEE, 2017, pp. 317-320.

32 J. F. Moulder, J. Chastain and R. C. J. King, Handbook of $x$-ray photoelectron spectroscopy: a reference book of standard spectra for identification and interpretation of XPS data, Perkin-Elmer Corporation, 1992, pp. 40-41.
33 K. Nakamoto, Infrared and Raman Spectra of Inorganic and Coordination Compounds: Part A: Theory and Applications in Inorganic Chemistry, WILEY, New Jersey, 6th edn, 2009, pp. 180-184.

34 J. Xu, G. Cheng, W. Yang, et al., Raman scattering from surface phonons in fine $\mathrm{Zn}$ particles coated with $\mathrm{ZnO}, J$. Phys. B: At., Mol. Opt. Phys., 1996, 29(24), 6227.

35 S. Kelly, F. H. Pollak and M. Tomkiewicz, Raman Spectroscopy as a Morphological Probe for $\mathrm{TiO}_{2}$ Aerogels, J. Phys. Chem. B, 1997, 101(14), 2730-2734.

36 G. J. Janz and D. W. James, Raman Spectra and Ionic Interactions in Molten Nitrates, J. Chem. Phys., 1961, 35(2), 739-745.

37 D. Shin and D. Banerjee, Enhancement of specific heat capacity of high-temperature silica-nanofluids synthesized in alkali chloride salt eutectics for solar thermal-energy storage applications, Int. J. Heat Mass Transfer, 2011, 54(56), 1064-1070. 